\section{Der Königsweg}

\author{
Reflektierte Praktiker Hin und wieder funktioniert Therapie \\ aus dem Bauch heraus. Verlassen sollte man sich darauf aber \\ nicht. Wer seinen Klienten eine zeitgemäße ergotherapeutische \\ Behandlung anbieten und sich professionell weiterentwickeln \\ will, kommt ums Reflektieren nicht herum.
}

$\rightarrow$

„Hätt’ ich doch nur!“ Manchmal kommt uns eine zündende Idee erst im Nachhinein. Zum Beispiel, wenn wir nach einer Therapieeinheit das unangenehme Gefühl haben: Das lief nicht optimal. Ein Gefühl, das regelrecht quälen kann. Aber auch dazu anregen, die Therapieeinheit noch einmal Revue passieren zu lassen und nach besseren Lösungen zu suchen. Dieser Reflexionsprozess erinnert daran, wie es ist, in einen Spiegel zu schauen: Es entstehen Bilder und Vorstellungen von sich selbst in einer konkreten Situation. Das „Spiegelbild“ zeigt Möglichkeiten auf, wie man sich angemessen verhalten und das eigene Vorgehen an wechselnde Anforderungen anpassen kann. Während dieser Selbsterkundung bewegen sich Ergotherapeuten auf einer Metaebene und folgen meist einer konkreten Vision davon, wie sie professionell arbeiten möchten [1]. Zum Beispiel klientenzentriert, evidenz- und handlungsbasiert. Indem sie ihre tatsächliche Vorgehensweise mit ihrer Idealvorstellung abgleichen, können sie Verbesserungsmöglichkeiten identifizieren und nutzen. Mit dem Ziel, sich professionell weiterzuentwickeln und effektiver zu handeln [1-3].

Direkt oder im Nachgang $\rightarrow$ Reflexion muss aber nicht erst nach der Handlung einsetzen. Ergotherapeuten können auch bereits während der Handlung - also im Moment der Ent- scheidungsfindung - über ihr eigenes Vorgehen nachdenken [3-5]. Zum Beispiel, wenn sie in einer Therapiesituation überlegen, wie sie klientenzentriert auf das Verhalten eines Klienten reagieren können. Oder wie sie ihre therapeutische Anleitung und Unterstützung am besten an seine veränderten Bedürfnisse anpassen. Dabei verknüpfen sie ihre früheren und aktuellen Erfahrungen mit ihrem theoretischen Wissen und greifen auf vielfältige Reasoning-Prozesse zurück, wie das pragmatische, konditionale, ethische oder narrative Reasoning [3, 5]. Immer mit dem Ziel, ihrem Klienten die bestmögliche Behandlung zukommen zu lassen. Denn eines ist in der Ergotherapie unbestritten: Es gibt keine Patentlösungen. Jede Situation ist einzigartig und erfordert damit eine einzigartige Herangehensweise. Das Reflektieren in und nach der Handlung ist somit grundlegend für eine effektive Praxis $[3,5,6]$.

\section{Komplexer Prozess $\rightarrow$}

Es gibt zahlreiche Versuche, die reflexive Praxis zu definieren. Einige Definitionen konzentrieren sich auf das Zusammenspiel von Denken und Handeln, das eine bessere Praxis ermöglichen soll. Andere betonen die Chance, aus Erfahrungen zu lernen und eine höhere Selbsterkenntnis zu erlangen. Oder sie betrachten die reflexive Praxis als Voraussetzung dafür, theoretische Kenntnisse systematisch in die Praxis umzusetzen und positive Ergebnisse
Das Reflektieren ist grundlegend für eine effektive Praxis. Es findet meist im Anschluss an eine Handlung statt oder bereits währenddessen, also direkt in der Entscheidungsfindung.

erzielen zu können [3, 4, 7-9]. Manche Autoren appellieren zudem an Ergotherapeuten, grundlegende Annahmen ihrer Profession kritisch zu reflektieren. Etwa die Frage, welchen Blick Ergotherapeuten auf Krankheit haben [10].

Um hier Licht ins Dunkel zu bringen und die Komplexität einer reflexiven Praxis zu beleuchten, hat die Ergotherapeutin Dr. Elizabeth Anne Kinsella sechs grundlegende Aspekte zusammengestellt [7]. Demnach versuchen reflektierte Ergotherapeuten ...

$\rightarrow$ aus ihren Erfahrungen zu lernen,

$\rightarrow$ die Quellen, Arten und Grenzen ihres Wissens zu erkennen,

$\rightarrow$ den Kontext der Klienten einzubeziehen,

$\rightarrow$ eigene Sichtweisen und Annahmen zu hinterfragen,

$\rightarrow$ Widersprüche zwischen ihrer „Philosophie der Praxis“ und deren Umsetzung zu erkennen und aufzulösen sowie

$\rightarrow$ Reflexionen und Aktionen ausgewogen in der Praxis zu vereinen.

Achtung: Forschung! $\rightarrow$ Nachdenken alleine reicht natürlich nicht aus, wenn man dem Klienten eine zeitgemäße ergotherapeutische 
„Indem wir unsere tatsächliche

Vorgehensweise mit unserer Idealvorstellung abgleichen, können wir Verbesserungsmöglichkeiten identifizieren."

„Durch Reflektieren entwickeln wir uns professionell weiter und handeln effektiver"

„Nachdenken alleine reicht nicht aus. Wir müssen auch den aktuellen Forschungsstand in unsere Reflexionsprozesse einbeziehen."

Behandlung anbieten will. Hierzu ist es auch nötig, den aktuellen Forschungsstand in die eigenen Reflexionsprozesse einzubeziehen. Sprich: evidenzbasiert zu arbeiten. Dabei gilt es, nach der bestverfügbaren wissenschaftlichen Evidenz zu suchen und diese mit der eigenen klinischen Expertise und den Werten des Klienten zu verknüpfen [11, 12]. Dann kann die evidenzbasierte Praxis ihren praktischen Nutzen voll entfalten: das gewählte Vorgehen wissenschaftlich zu untermauern und positive Outcomes zu generieren [3]. Außerdem können sich Ergotherapeuten von Forschungsergebnissen inspirieren lassen und so ihre Denk- und Herangehensweisen optimieren. Etwa, indem sie tiefere Einblicke in das Erleben betroffener Klienten erhalten oder auf geeignetere Assessments und Interventionen aufmerksam werden. Vorausgesetzt natürlich, sie bringen die Bereitschaft und Offenheit mit, vertraute Pfade zu verlassen und neue Wege auszuprobieren [3].

Gemeinsam geht's leichter $\rightarrow$ Die Welt ist prinzipiell unsicher. So sieht es Donald Schön, der den Begriff des reflektierten Praktikers maßgeblich geprägt hat. Daher sollten Ausbildungs- und Studienangebote angehende Ergotherapeuten darauf vorbereiten, mit dieser Unsicherheit umzugehen [4, 13, 14]. Um den Anforderungen der Praxis gerecht zu werden, müssen sie vor allem lernen, ihr theoretisches Wissen auf die Belange des einzelnen Klienten abzustimmen, Erkenntnisse aus der Praxis heraus zu entwickeln und wieder an der Praxis zu überprüfen [13]. Ein dynamischer Prozess, den Praxisanleiter, Kollegen, Mentoren und Supervisoren unterstützen können [5, 10, 15-17].

Nicht nur angehende Ergotherapeuten profitieren von einem solchen Austausch, sondern auch erfahrene Berufspraktiker. Insbesondere, wenn sie in einem konkreten Fall an ihre Grenzen stoßen. Arbeiten sie in einem gut funktionierenden Team, können sie Probleme bereits ansprechen, bevor sie eine Lösung gefunden haben $[17,18]$. Etwa im Rahmen einer kollegialen Beratung oder reflexiven Peer-Gruppe.

Solche systematischen Formen des Austauschs können nachweislich dazu beitragen, berufliche Beanspruchung zu vermindern, Problemlösefertigkeiten und Zufriedenheit zu steigern sowie das professionelle Vorgehen zu verbessern [15, 17, 19-22]. Erlebte Probleme gelten dabei nicht als Zeichen der Unzulänglichkeit. Sondern als Möglichkeit, vorhandene Schwellen zu überqueren, neue Wege zu entdecken und sich weiterzuentwickeln.

Florence Kranz

$\mathbf{a}$ Literaturverzeichnis

www.thieme-connect.de/products/ergopraxis $>$ „Ausgabe 7-8/16“

\section{Autorin}

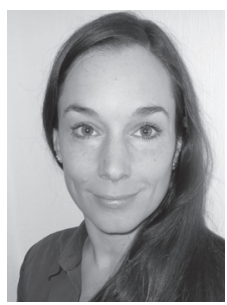

Florence Kranz, Ergotherapeutin $\mathrm{BcOT}(\mathrm{NL})$ und M.A. Gesundheitsmanagement, arbeitet als freie Journalistin, Redakteurin und Dozentin. Seit 2010 schreibt sie regelmäßig Beiträge für ergopraxis. 